\title{
Computer Aided Design and Simulation of a Multiobjective Microstrip Patch Antenna for Wireless Applications
}

\author{
Chitra Singh $^{1^{*}}$ and R. P. S. Gangwar ${ }^{2}$ \\ ${ }^{1,2}$ Department of Electronics \& Communication Engineering, College of Technology, \\ G.B.Pant Univ. of Agric. \& Tech., \\ Pantnagar, Uttarakhand, India. \\ *chitra.singh88@gmail.com
}

\begin{abstract}
The utility and attractiveness of microstrip antennas has made it ever more important to find ways to precisely determine the radiation patterns of these antennas. Taking benefit of the added processing power of today's computers, electromagnetic simulators are emerging to perform both planar and 3D analysis of high-frequency structures. One such tool studied was IE3D, which is a program that utilizes method of moment. This paper makes an investigation of the method used by the program, and then uses IE3D software to construct microstrip antennas and analyze the simulation results. The antenna offers good electrical performance and at the same time preserves the advantages of microstrip antennas such as small size, easy to manufacture as no lumped components are employed in the design and thus, is low cost; and most importantly, it serves multiple wireless applications.
\end{abstract}

Keywords- Electromagnetic simulation; microstrip antenna; radiation pattern; IE3D;

\section{INTRODUCTION}

Microstrip antennas have become increasingly popular for microwave and millimeter wave applications, because they offer several distinct advantages over conventional microwave antennas. These advantages include small size, easy to fabricate, lightweight, and conformability with the hosting surfaces of vehicles, aircraft, missiles, and direct integration with the deriving electronics. Microstrip antennas are assigned different names, such as, printed antennas (can be printed directly onto a circuit board), planar antennas, microstrip patch antennas or simply microstrip antennas (MSA). MSA in general consists of radiating conducting patch, a conducting ground plane, a dielectric substrate sandwiched between the two, and a feed connected to the patch through the substrate. A patch radiates from fringing fields around its edges as shown in Fig. 1. As can be seen in the figure, the electric field does not stop abruptly near the patch's edges: the field extends beyond the outer periphery.

These 'field extensions' are known as 'fringing fields' and cause the patch to radiate.

Their utility and increasing demand makes it necessary to develop a precise method of analyzing these types of antennas. Several theoretical methods of determining far field patterns have been formulated, such as the aperture method, the cavity method, modal method and the transmission line method [1-3].
Although these methods are capable of providing a physical insight, are not as accurate as some numerical techniques can be because all these methods contain approximations. As fullwave solutions are more accurate and applicable to many structures, some researchers begin to study the MoM-based solution for MSAs, such as [4-13] in recent years. The integral equation formulation solved using the method of moments (MoM) [14-16] is known as an efficient tool. The application of the MoM to a planar configuration results in a dense coupling matrix. This matrix can be considered as the coefficients matrix of a few systems of linear equations which are required to be solved. With the increasing capability of today's computers, numerical techniques offer a more accurate reflection of how microstrip antennas actually perform. Their use reduces the need to modify the final dimensions using a knife to remove metal or metal tape to increase the patches.

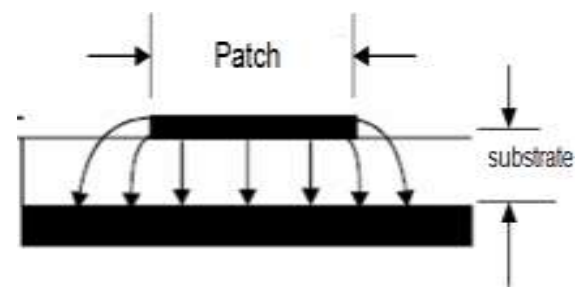

Fig. 1 A patch radiating from fringing fields around its edges

The MoM modeling program used in this paper is IE3D full wave EM solution of Zeland simulation software. IE3D is the computational power-house that when given an input file performs all the calculations necessary to obtain the fields and/or currents at each grid point in the model. Full wave EM simulation refers to the solution of Maxwell's or differential equations governing macro electromagnetic phenomenons, without assuming frequency at DC or any other assumptions. Full-wave EM simulations are very accurate from very low frequency to very high frequency.

The paper is organized in the following way. Section II presents an explanation of the method of moments as a solution to the integral equations. In Section III, the proposed microstrip antenna designing procedure and its geometry is described and discussed. In Section IV, the IE3D full wave EM solution of Zeland simulation software based on MoM modeling program is employed to construct a unique microstrip patch antenna, and 
simulation results are presented and discussed. Finally, conclusions are drawn in Section V.

\section{General Description OF THE METHOD OF ANALYSIS USED}

The integral equations are formulated with a full dyadic Green's function and the matrix elements are computed completely numerically in the spatial domain [3]. IE3D can model truly arbitrary 3D metal structures. The method of moments (MOM) [17] expands the currents on an antenna (or scattering object) in a linear sum of simple basis functions. The approximate solution is a finite series of these basis functions [18]:

$$
f_{a}=\sum_{i=1}^{N} a_{i} f_{i}
$$

Coefficients are obtained by solving integral equations satisfying boundary conditions on the surface of the antenna (or object). The integral equation can be expressed in the form $L f_{a}$ $=g$, where $L$ is a linear operator, usually a scalar product using an integral, $f_{a}$ the unknown currents given by Eq. (1), and $g$ the known excitation or source function. The summation of Eq. (1) is substituted into the linear operator equation and scalar product integral is used to calculate the terms in a matrix equation. The solution of the matrix equation determines the coefficients of current expansion. The MOM produces filled matrices. The art of the MOM is in choosing basis functions and deriving efficient expressions for evaluating the fields using the basis function currents. Common basis functions are simple staircase pulses, overlapping triangles, trigonometric functions, or polynomials. The method does not satisfy boundary conditions at every point, only over an integral average on the boundaries. By increasing the number of basis functions, the method will converge to the correct solution. Spending excessive time on the solution cannot be justified if it greatly exceeds our ability to measure antenna performance accurately using real hardware [18].

This approach is relevant to a general planar geometry in stack or multi-layer configuration. To highlight the main idea, general description for a conducting patch on top of a single dielectric layer with a ground plate on bottom is being presented, without loss of generality, as shown in Fig. 2.
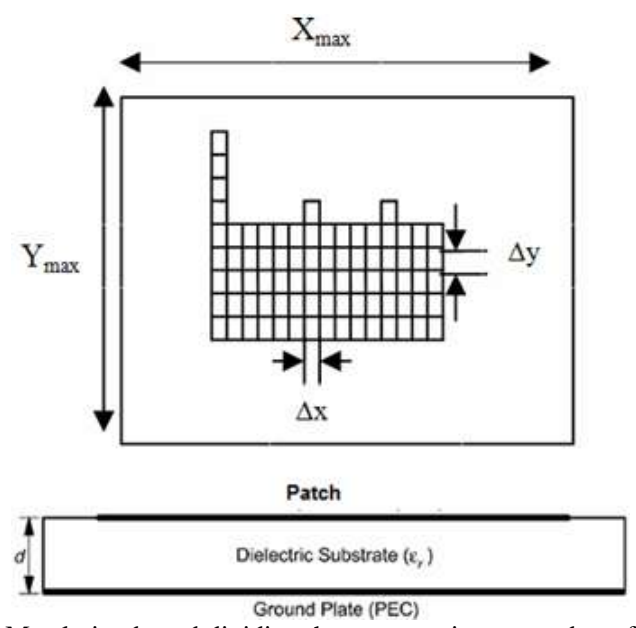

Fig. 2 MoM solution by subdividing the structure into a number of segments
Here $\varepsilon_{r}$ is dielectric constant of the substrate and $d$ is its thickness. Method of moments (MoM) is used to solve the problem and analyze the patch antenna performance. For brevity significant steps are presented here. As shown in Fig. 2, the structure is subdivided into a number of segments in first step. In second step, the unknown induced current on the structure is expanded in terms of known basis functions weighted by unknown amplitudes. The excitation current at the source locations is modeled using another set of basis functions [19].

$$
\begin{aligned}
& J_{x}^{i}(x, y)=\sum_{n=1}^{N_{x}^{i}} A_{n}^{i} E T\left(x-a_{n}^{i}\right) R\left(y-b_{n}^{i}\right) \\
& J_{y}^{i}(x, y)=\sum_{n=1}^{N_{y}^{i}} B_{n}^{i} E T\left(x-c_{n}^{i}\right) E T\left(y-d_{n}^{i}\right) \\
& J_{x}^{e}(x, y)=\sum_{n=1}^{N_{x}^{e}} A_{n}^{e} O T\left(x-a_{n}^{e}\right) R\left(y-b_{n}^{e}\right) \\
& J_{y}^{e}(x, y)=\sum_{n=1}^{N_{y}^{e}} B_{n}^{e} R\left(x-c_{n}^{e}\right) O T\left(y-d_{n}^{e}\right)
\end{aligned}
$$

where the superscripts $i$ and $e$ mean induced and excitation currents, respectively. $J_{x}$ and $J_{y}$ are the $x$ and $y$ components of the current, respectively. $N_{x}$ and $N_{y}$ are the numbers of $x$ and $y$ directed basis functions. $A_{n}$ and $B_{n}$ are the amplitudes of the $n$th $x$ and $y$ directed basis functions, respectively. $a_{n}$ and $b_{n}$ are the $x$ and $y$ coordinates of the center of the $n$th $x$ directed basis function. $c_{n}$ and $d_{n}$ are the $x$ and $y$ coordinates of the center of the $n$th $y$ directed basis function, respectively. The even triangular, $E T$, odd triangular, $O T$, and rectangular, $R$, functions are defined as follows[19]:

$$
\begin{aligned}
& \operatorname{ET}(u)=\left\{\begin{array}{lc}
\frac{\left(u+\Delta_{u}\right)}{\Delta_{u}^{2}} ; & -\Delta_{u}<u<0 \\
\frac{\left(\Delta_{u}-u\right)}{\Delta_{u}^{2}} ; & 0<\mathrm{u}<\Delta_{u} \\
0 ; & \text { otherwise }
\end{array}\right\} \\
& \text { OT }(u)=\left\{\begin{array}{ll}
-\frac{\left(u+\Delta_{u}\right)}{\Delta_{u}^{2}} ; & -\Delta_{u}<u<0 \\
\frac{\left(\Delta_{u}-u\right)}{\Delta_{u}^{2}} ; & 0<\mathrm{u}<\Delta_{u} \\
0 ; & \text { otherwise }
\end{array}\right\}
\end{aligned}
$$




$$
R(u)=\left\{\begin{array}{lc}
\frac{1}{\Delta_{u}} ; & -\frac{\Delta_{u}}{2}<u<\frac{\Delta_{u}}{2} \\
0 ; & \text { otherwise }
\end{array}\right\}
$$

where the variable $u$ is replaced by either $x$ or $y, \Delta_{\mathrm{u}}$ is the width of the segment along the $u$ direction. Boundary conditions are satisfied in third step of MoM solution procedure [18]. Assuming both, the patch and ground as perfect conductors, the tangential electric field should vanish. Expressing this boundary condition in terms of an integral equation and substituting the previous current expansion into this integral equation and applying Galerkin's testing, results in the

$$
\left[\begin{array}{l}
{\left[E_{x}\right]} \\
{\left[E_{y}\right]}
\end{array}\right]=\left[\begin{array}{ll}
{\left[Z_{x x}^{i}\right]} & {\left[Z_{x y}^{i}\right]} \\
{\left[Z_{y x}^{i}\right]} & {\left[Z_{y y}^{i}\right]}
\end{array}\right]\left[\begin{array}{l}
{\left[A^{i}\right]} \\
{\left[B^{i}\right]}
\end{array}\right]+\left[\begin{array}{ll}
{\left[Z_{x x}^{e}\right]} & {\left[Z_{x y}^{e}\right]} \\
{\left[Z_{y x}^{e}\right]} & {\left[Z_{y y}^{e}\right]}
\end{array}\right]\left[\left[\begin{array}{l}
\left.A^{e}\right] \\
{\left[B^{e}\right]}
\end{array}\right]=\left[\begin{array}{l}
{[0]} \\
{[0]}
\end{array}\right]\right.
$$

following matrix equation:

where the elements of the vectors $\left[E_{y}\right]$ and $\left[E_{x}\right]$ are the total $y$ and $x$ components of the electric field at the test points. Thus, knowing the impedance submatrices, and the amplitudes of the excitation current $\left[A^{e}\right]$ and $\left[B^{e}\right]$, and, the unknown amplitudes of the induced current, $\left[A^{i}\right]$ and $\left[B^{i}\right]$ can be calculated. A number of distributions of the excitation current can be imposed and corresponding to each distribution, the induced current can be obtained from (9) by solving a system of linear equations. As a last step, a de-embedding technique is employed to obtain the S-parameters matrix which characterizes the structure. Some de-embedding techniques are given in [19]. Filling of the submatrices : $\left[Z_{x x}^{i}\right],\left[Z_{x y}^{i}\right],\left[Z_{y x}^{i}\right]$ and $\left[Z_{y y}^{i}\right]$ takes most of the time in electrically small and medium size structures. However, IE3D is efficient full wave electromagnetic simulation software which solves the above matrices for unknown surface currents and computes the important parameters, such as, return loss, voltage standing wave ratio, gain, directivity, radiation pattern etc. [21].

\section{ANTENNA GeOMETRY AND DESIGN PROCEDURE}

An effort to provide multiple functionality, usually results in increased size and additional complexity. For example, use of stacked patches/ multiple layers in reference [22], [23] and [24] increase the volume of antenna. Using patch antenna closely surrounded by patches occupies more lateral area. Similarly, air substrate has been widely used which increases thickness and thus, volume of the antenna. Approaches to enhance bandwidth include increasing substrate thickness, using electrically thick elements and parasitic element either in co-planar or stack configuration. But all these techniques increase the complexity and the size of the antenna. In our design, focus was on obtaining good electrical performance without design complexity or increased overall volume.

Physical layout of proposed antenna is given in Fig. 3. Slots of appropriate shape are incorporated on patch to perturb the surface current distribution, so as to optimize the results while maintaining single layer. The patch is shorted with the ground using a bent shorting plate on right edge. The proposed antenna's performances are analyzed using MoM based IE3D full wave electromagnetic simulation software. Since its formal introduction in 1993 IEEE International Microwave Symposium (IEEE IMS 1993), the IE3D has been adopted as an industrial standard in planar and 3D EM simulation. Since then, much improvement has been achieved in IE3D.

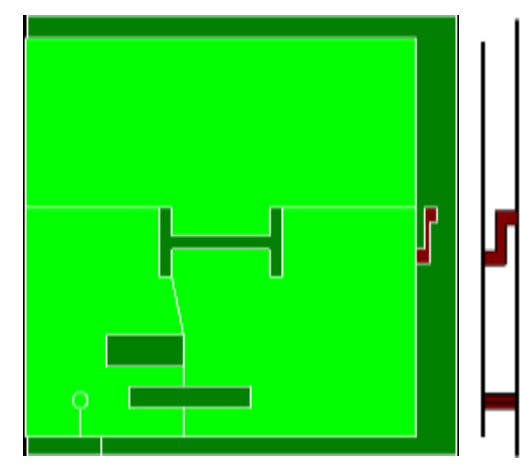
(a) Top view
Fig. 3 Structure of the proposed antenna
(b) Side view

\section{RESUlTS AND Discussion}

The simulator tool computes useful quantities of interest such as radiation pattern, input impedance, gain etc. Effect of shorting plates and slots can be well understood from the current density distribution as shown in Fig. 4 for different frequencies. The probe feed at one end provides current source which flows through the shorting plate to the bottom conducting plate. However, in between the feed and plate, slots are incorporated in centre with four branches to provide more and more perturbation to the surface current density distribution.

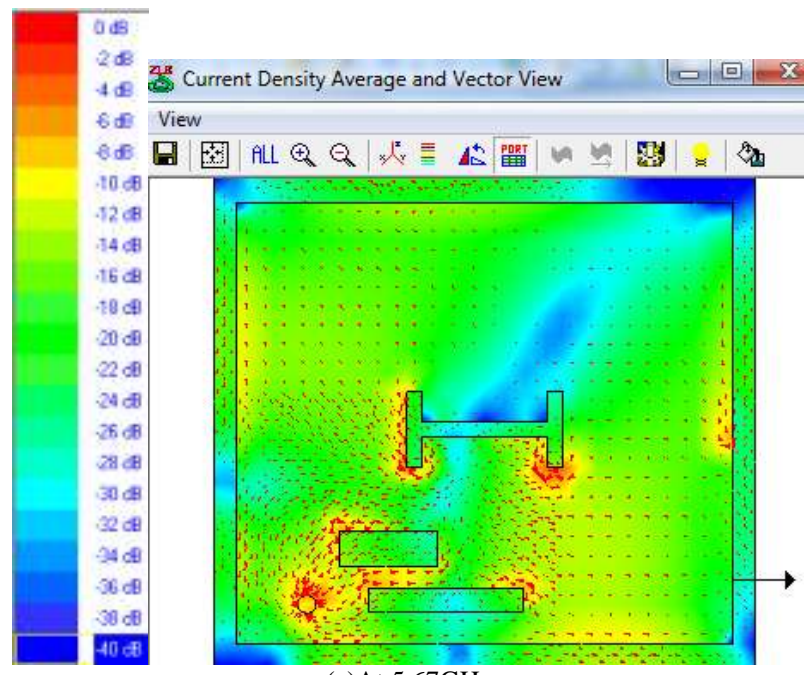

(a) $\mathrm{At} 5.67 \mathrm{GHz}$ 


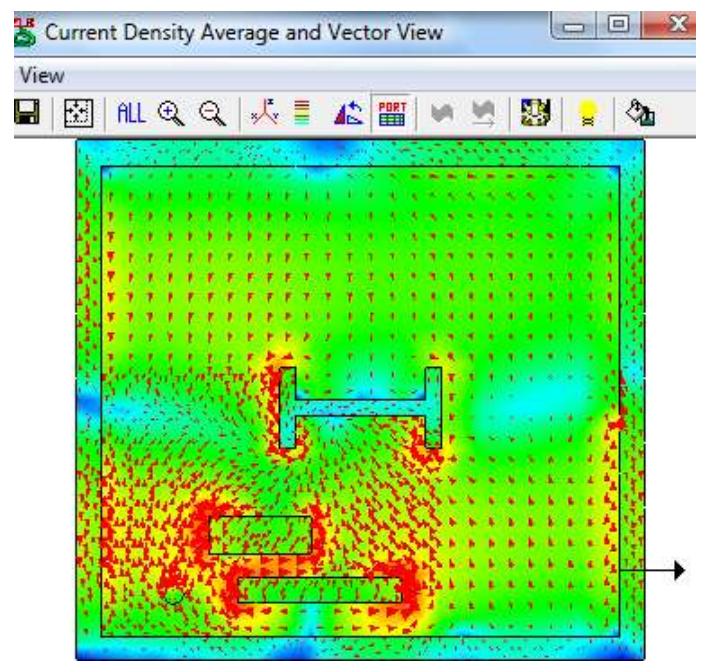

(b)At $6.12 \mathrm{GHz}$

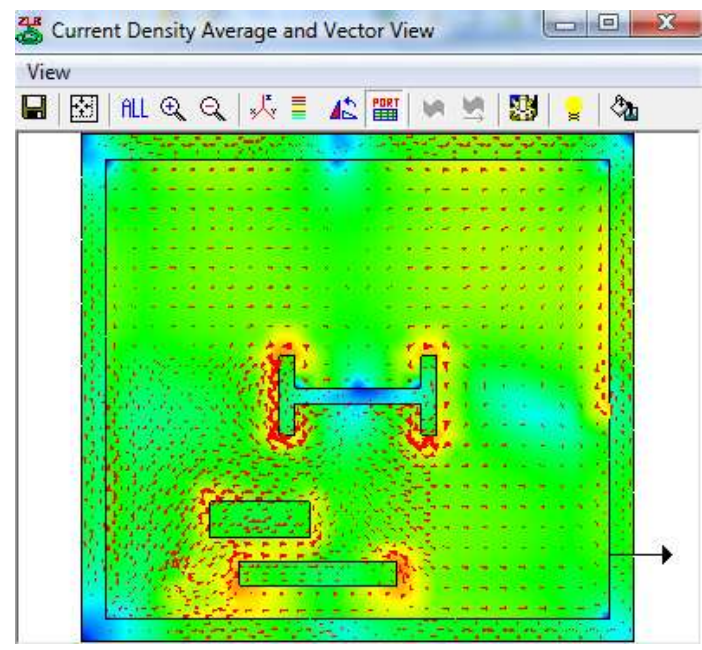

(c) At $6.386 \mathrm{GHz}$

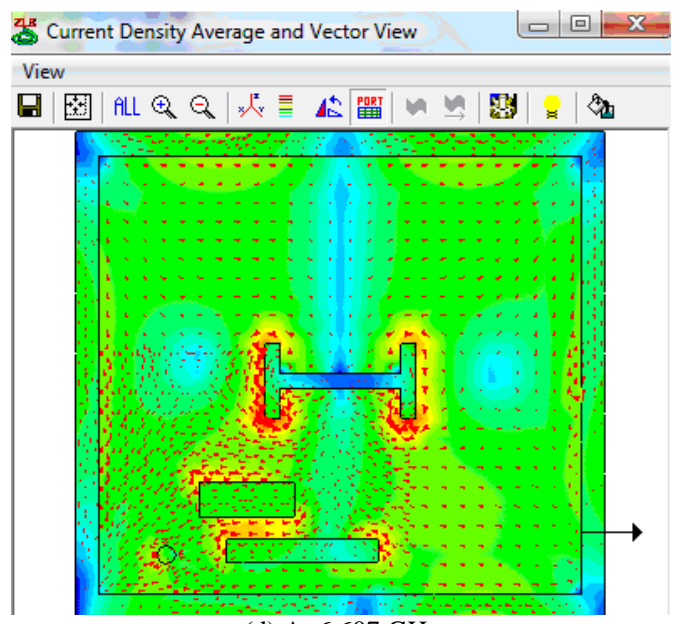

(d) At $6.607 \mathrm{GHz}$

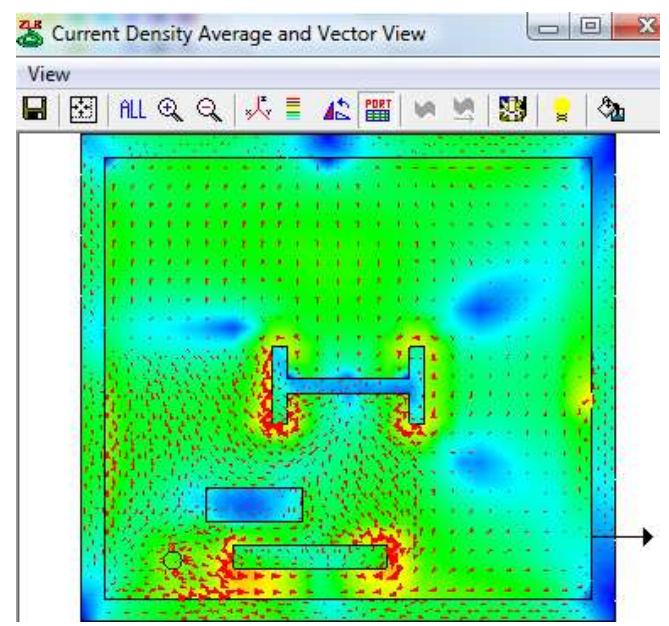

(e) At $7.759 \mathrm{GHz}$

Fig. 4 Current density distribution over the antenna surface

Fig. 5(a) introduces return loss vs. frequency curve for the proposed design. Return loss is defined as the ratio of the Fourier transforms of the incident pulse and the reflected signal. The bandwidth can be obtained from the return loss (RL) plot. The bandwidth is said to be those range of frequencies over which the return loss is less than $-10 \mathrm{~dB}$, which is approximately equivalent to a voltage standing wave ratio (VSWR) of less than 2:1. The simulated results show five bands at $5.673 \mathrm{GHz}, 6.463 \mathrm{GHz}, 6.880 \mathrm{GHz}, 7.686 \mathrm{GHz}$ and $8.402 \mathrm{GHz}$. The first band provides Wi-MAX, WLAN (IEEE 802.11a standard) and ISM (Industrial, Scientific and Medical) band applications. These days, WLAN is also being considered for mobile phone applications. Next three bands are useful for various wireless applications of $\mathrm{C}$-Band, such as, object identification for surveillance applications, location tracking applications operating in the frequency band from $6 \mathrm{GHz}$ to 9 $\mathrm{GHz}$, cordless phones and other airborne applications. The last band offers some $\mathrm{X}$-band applications like, ground-probing and wall-probing radar, tank level probing radar, sensors, automotive radar etc. 
$\rightarrow-\Gamma \mathrm{dB}[\mathrm{S}(1,1)]$

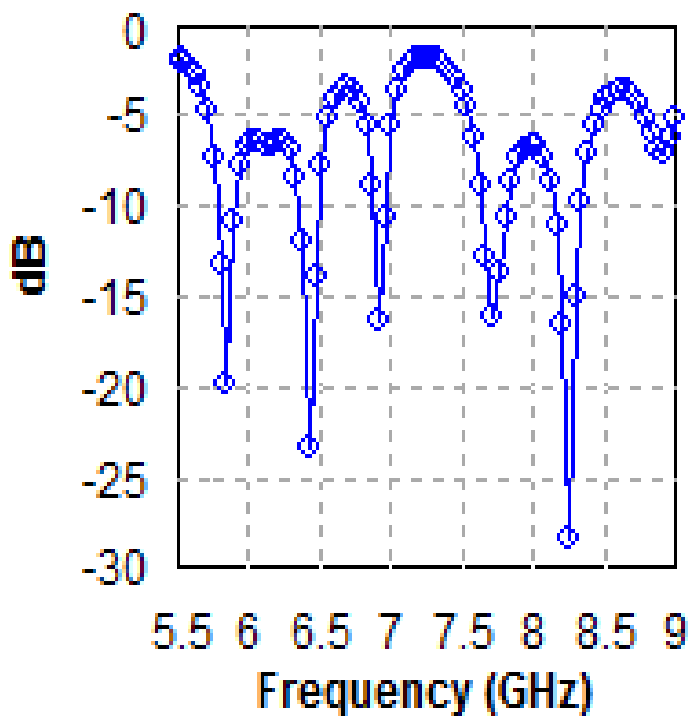

(a)

Total Field Gain vs. Frequency

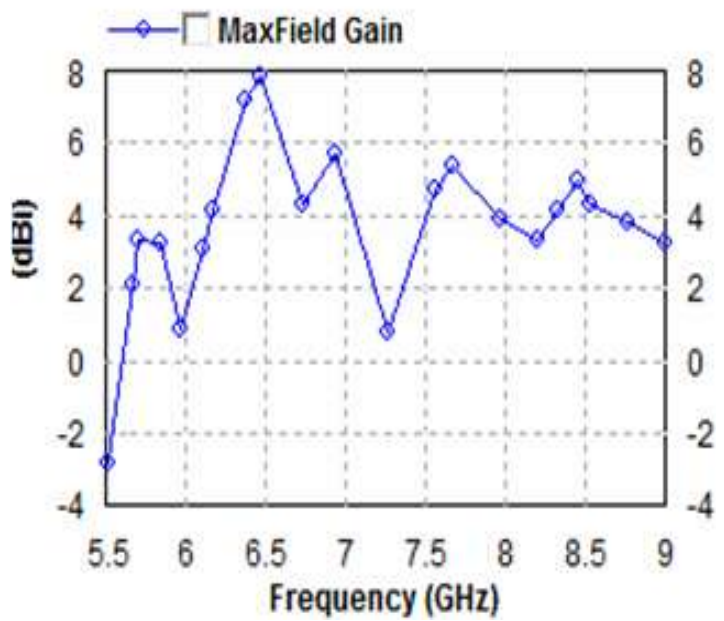

(b)
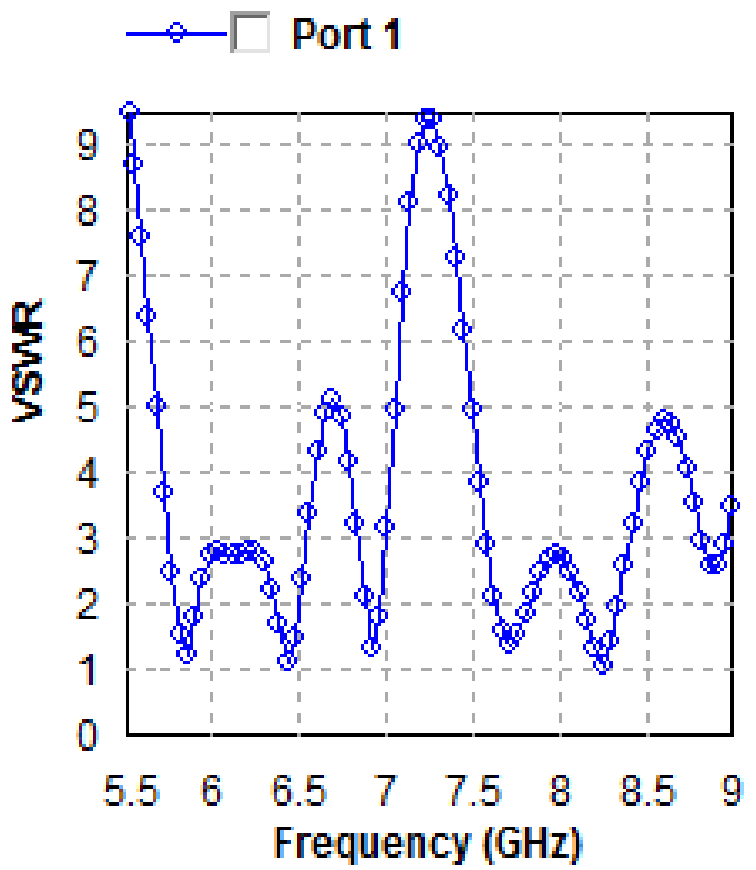

(c)

Field Directivity vs. Frequ

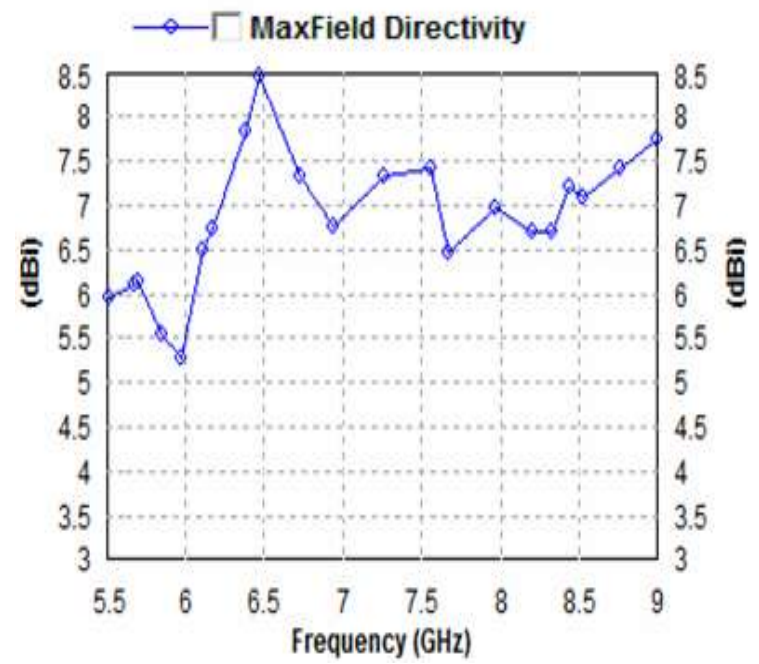

(d)

Fig. 5 (a) Return loss plot, (b) Gain, (c) VSWR, and (d) Directivity vs frequency plot 


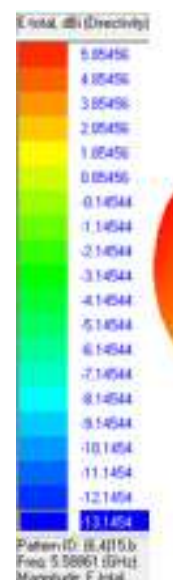

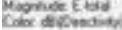

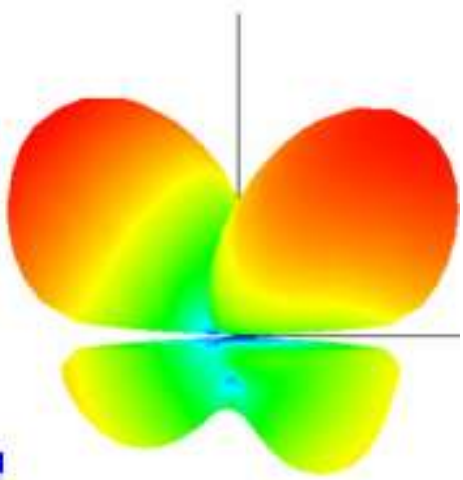

(a) At $5.588 \mathrm{GHz}$

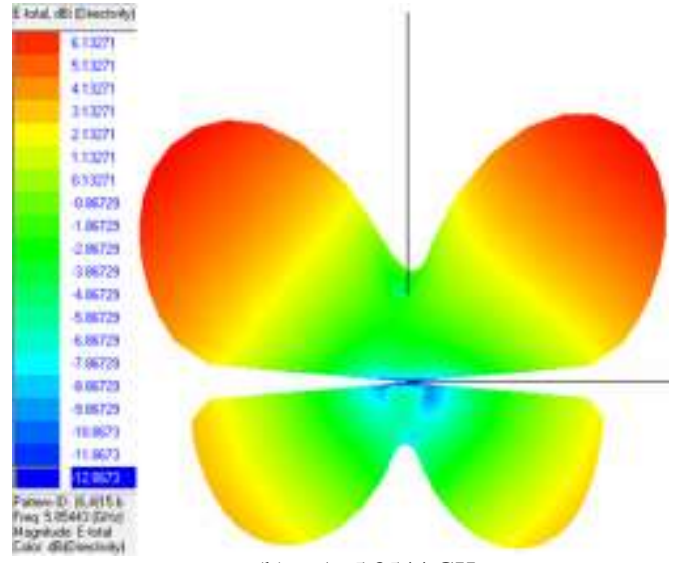

(b) At $5.8544 \mathrm{GHz}$

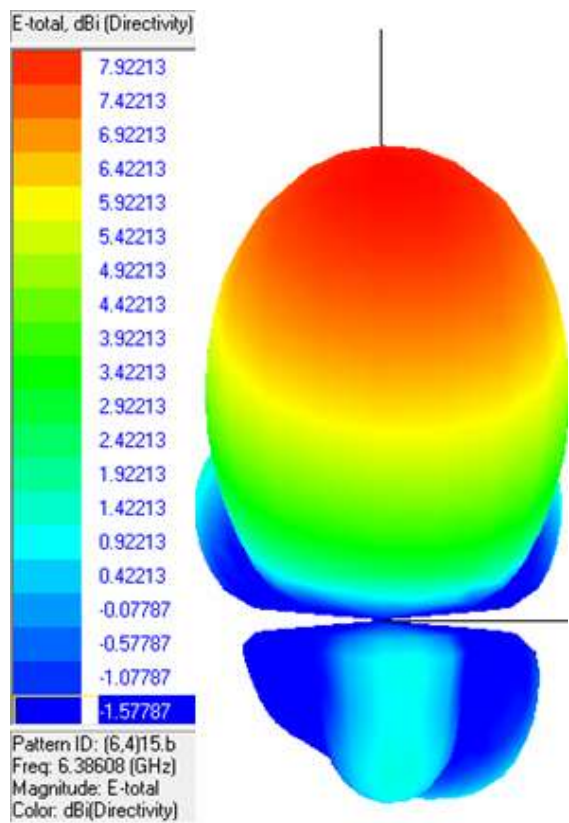

(c) At $6.38 \mathrm{GHz}$

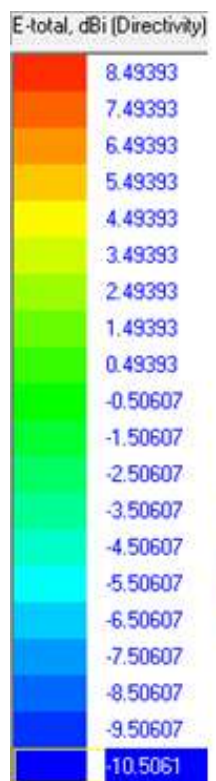

Pattern 1D: 16,415 b Freq $6.60759(\mathrm{GHz})$ Magnitude: E-total Color: dBi(Directivity)

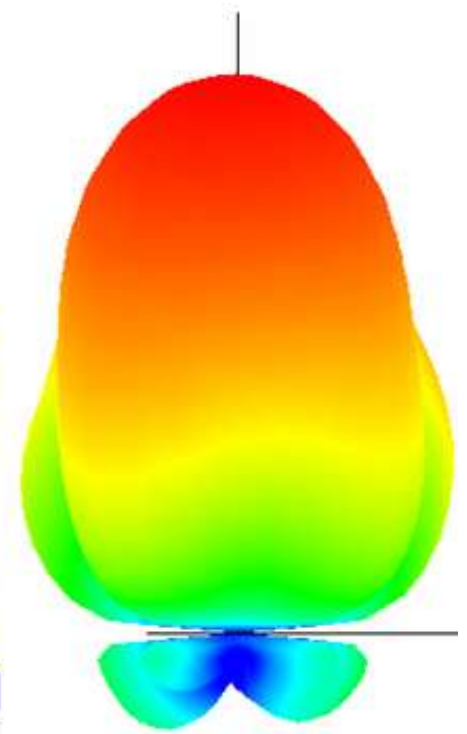

(c) At $6.607 \mathrm{GHz}$

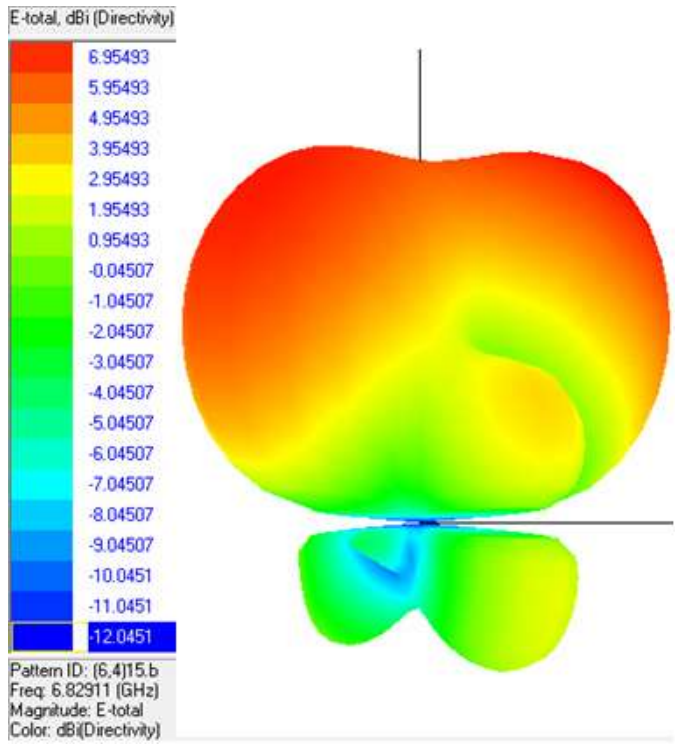

(e) At $6.829 \mathrm{GHz}$ 


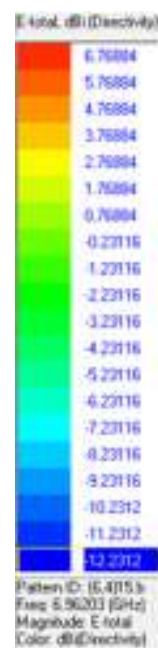

Etotal d8i (Directivity)

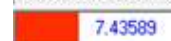

6.43589

5.43589

4.43589

3.43589

243589

1.43589

0.43589

$-0.56411$

$-1.56411$

.256411

$-3.56411$

$-4.56411$

5.56411

.656411

.7 .56411

$-8.56411$

.9 .56411

$-10.5641$

11.5641

(g) At $7.537 \mathrm{GHz}$

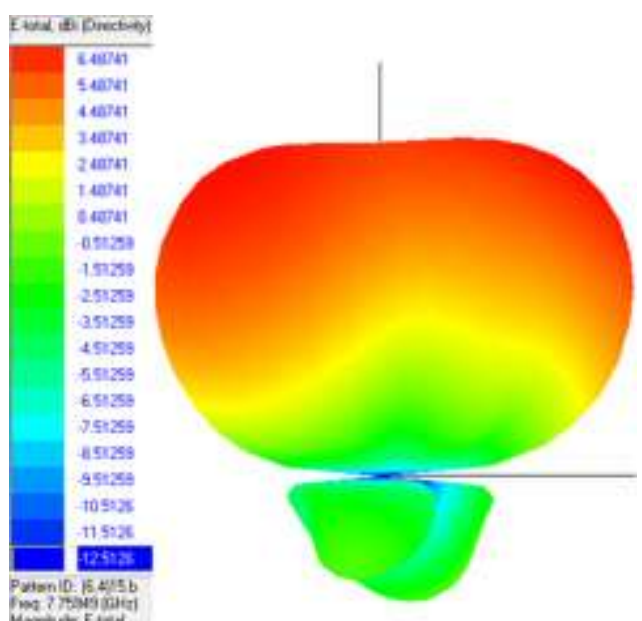

(h) At $7.759 \mathrm{GHz}$

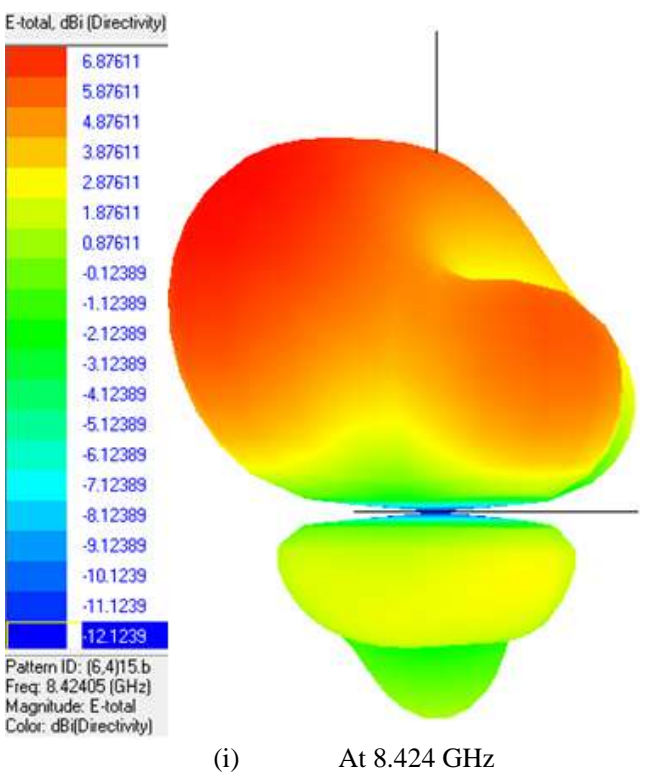

Fig. 6 True 3D radiation patterns on IE3D software

The antenna achieves a very high simulated directivity and gain as shown in Fig. 5(d) and 5(b). The peak gain reaches 8 dBi. Fig. 6 introduces simulated true 3D simulated radiation patterns at different frequencies.

\section{CONCLUSION}

It was shown that the method as used by IE3D can be used to efficiently model microstrip antennas. With computers getting larger, faster, and cheaper every month, large models will be able to be simulated with little difficulty. The rapid growth of wireless communications demands that antennas be low profile and allow operation at multiple frequency bands, eliminating the need for separate antennas for each application. This paper first presents a brief explanation of the method of moment. Then a compact, multipurpose and low manufacturing cost microstrip patch antenna is designed using this software and the simulation results are presented. The designed antenna possesses very high directivity and peak gain. According to the good electrical performance over the frequency range of interest, the proposed antenna should find use for several wireless applications like, Wi-MAX, WLAN, probing and automotive radar, wireless sensors, and various ground based and airborne applications in C-band.

\section{REFERENCES}

[1] Balanis C.A., "Antenna Theory-Analysis and Design"

[2] K.R.Carver,J.W. Mink "Microstrip Antenna Technology". IEEE Trans. Antenna Propagat., Vol AP-29, No. 1 pp. 2-24.January 1981.

[3] R. Garg and P. Bhartia, Microstrip antenna handbook. Boston: Artech house, 2001

[4] Jin Sun, Chao-Fu Wang, Le-Wei Li, Mook-Seng Leong, "Further Improvement for Fast Computation of Mixed Potential Green's Functions for Cylindrically Stratified Media". IEEE Transactions Antennas Propogat, vol.52, pp.3026- 3036, 2004.

[5] S. Karan, V.B.Ertürk, and A. Altintas, "Closed-Form Green's Function Representations in Cylindrically Stratified Media for Method of Moments Applications”. IEEE Transactions Antennas Propogat, vol.57, pp.1158-1168, 2009. 
[6] V.B.Ertürk and R.G.Rojas, "Efficient Computation of Surface Fields Excited on a Dielectric-Coated Circular Cylinder". IEEE Transactions Antennas Propogat, vol.48, pp.1507-1516, 2000.

[7] V.B.Ertürk and R.G.Rojas, "Paraxial Space-Domain Formulation for Surface Fields on a Large Dielectric Coated Circular Cylinder". IEEE Transactions Antennas Propogat, vol.50, pp.1577-1587, 2002.

[8] V.B.Ertürk and R.G.Rojas, "Efficient Analysis of Input Impedance and Mutual Coupling of Microstrip Antennas Mounted on Large Coated Cylinders". IEEE Transactions Antennas Propogat, vol.51, pp.739-749, 2003.

[9] T.N.Kaifas and J.N.Sahalos, "Analysis of Printed Antennas Mounted on a Coated Circular Cylinder of Arbitrary Size". IEEE Transactions Antennas Propogat, vol.54, pp.2797-2807, 2006.

[10] Mang.He and Xiaowen.Xu, "Closed-Form Solutions for Analysis of Cylindrically Conformal Microstrip Antennas with Arbitrary Radii”. IEEE Transactions Antennas Propogat, vol.53, pp.518-525, 2005.

[11] A. Svezhentsev, G.A.E. Vandenbosch, "Effcient Spatial Domain Moment Method Solution of Cylindrical Rectangular Microstrip Antennas". IEE Proc.-Microw. Antennas Propag, vol.153, pp.376-384, 2006.

[12] R.Cüneyt Acar, "Mutual Coupling of Printed Elements on a Cylindrically Layered Structure Using Closed-Form Green's Functions". Progress in Electromagnetics Research, PIER 78, pp.103-127, 2008.

[13] C.Tokgöz and G.Dural, "Closed-Form Green's Functions for Cylindrically Stratified Media". IEEE Transactions Microwave Theory Tech, vol.48, pp.40-49, 2000.

[14] J. R. Mosig and F. E. Gardiol, "General integral equation formulation for microstrip antennas and scatters," Proc. Inst. Elect. Eng., pt. H, vol. 132, pp. 424-432, Dec. 1985.
[15] K. A. Michalski and D. Zheng, "Electromagnetic scattering and radiation by surfaces of arbitrary shape in layered media-Part I: Theory," IEEE Trans. Antennas Propagat., vol. 38, pp. 335-344, Mar.1990.

[16] I. Park, R. Mittra, and M. I. Aksun, "Numerically efficient analysis of planar microstrip configurations using closed-form Green's functions," IEEE Trans. Microwave Theory Tech., vol. 43, pp. 394-400, Feb. 1995.

[17] R.F.Harrington,FieldComputation by Moment Methods, Macmillan, New York, 1968; reprinted by IEEE Press, New York, 1993.

[18] Thomas A. Milligan, Modern Antenna Design, IEEE Press, A John Wiley $\&$ Sons, Inc., Publication, $2^{\text {nd }}$ Edition

[19] I. Park, R. Mittra, and M. I. Aksun, "Numerically efficient analysis of planar microstrip configurations using closed-form Green's functions," IEEE Trans. Microwave Theory Tech., vol. 43, pp. 394-400, Feb. 1995.

[20] M. I. Aksun, "A robust approach for the derivation of closed-form Green's functions," IEEE Trans. Microwave Theory Tech., vol. 44, pp. 651-658, May 1996.

[21] IE3DUser's Manual,Zeland Software,Inc.,Fremont. C

[22] B. Sanz-Izquierdo, J.C. Batchelor, R.J. Langley and M.I. Sobhy, "Single and double layer planar multiband PIFAs", EEE Transactions on Antennas and Propagation, vol. 54, pp. 1416-1422, 2006.

[23] S. D. Targonski, R. B. Waterhouse, and D. M. Pozar, "Design of wideband aperture-stacked patch microstrip antennas," IEEE Trans. Antennas Propagat., vol. 46, pp. 1245-1251, Sept. 1998.

[24] W.Chen, K. F.Lee, and R. Q. Lee, "Spectral-domain moment method analysis of coplanar microstrip parasitic subarrays," Microw. Opt. Technol. Lett., vol. 6, no. 3, pp. 157-163, 1993. 